\title{
ANÁLISE DA PRODUÇÃO CIENTÍFICA BRASILEIRA SOBRE AVALIAÇÃO PSICOLÓGICA EM ORIENTAÇÃO PROFISSIONAL
}

\author{
Rodolfo Augusto Matteo Ambiel \\ Psicólogo, mestre e doutorando pelo Programa de Pós-graduação Stricto Sensu em Psicologia da \\ Universidade São Francisco campus Itatiba e docente de cursos de graduação e pós-graduação \\ Lato Sensu de Psicologia da mesma universidade. Consultor externo de pesquisa da Editora Casa \\ do Psicólogo.

\begin{abstract}
Mariana Fralleti de Polli
Estudante do $10^{\circ}$ Semestre de Psicologia da Universidade São Francisco campus Itatiba.
\end{abstract}

\section{Resumo}

O presente trabalho objetivou a realização de um levantamento da literatura científica brasileira, publicada em bases de dados nacionais de periódicos científicos, a respeito da avaliação psicológica no contexto da orientação profissional. Para tanto, foi realizada uma busca nas bases PePSIC e Scielo em agosto de 2011, a partir de critérios de seleção e exclusão e termos definidos a priori, tendo como escopo de análise um total de 24 artigos que tiveram seus textos completos analisados. Os resultados foram organizados em três blocos, relativos aos autores, periódicos e aspectos metodológicos. São discutidos principalmente aspectos referentes aos estados de origem dos autores, as relações entre objetivos traçados e metodologias adotadas nos artigos e a aparente causalidade existe entre quantidade de publicações de artigos e aprovação para utilização profissional dos testes.

Palavras-chave: orientação vocacional, testes psicológicos, pesquisa científica.

\section{BRAZILIAN SCIENTIFIC PRODUCTION ANALYSIS ABOUT PSYCHOLOGICAL ASSESSMENT IN CAREER GUIDANCE}

\begin{abstract}
This study performed a survey of Brazilian scientific literature, published in national databases of scientific journals about the psychological assessment in the context of career guidance. To this end, a search was performed on the basis PePSIC and Scielo in August 2011, with selection and exclusion criteria and terms defined a priori, with the scope of analysis a total of 24 articles that were full texts analyzed. The results were organized into three blocks, about authors, journals and methodological aspects. Issues related to states of origin of the authors, the relationship between goals set and methods adopted in the articles and the apparent causation between number of publications of articles for professional use and approval of the tests are discussed mainly.
\end{abstract}

Keywords: career guidance, psychological tests, scientific research. 


\title{
ANÁLISIS DE LA PRODUCCIÓN CIENTÍFICA BRASILEÑA DE EVALUACIÓN PSICOLÓGICA EN ORIENTACIÓN PROFESIONAL
}

\begin{abstract}
Resumen
Este trabajo realiza un estudio de la literatura científica brasileña, publicada en bases de datos nacionales de las revistas científicas acerca de la evaluación psicológica en el contexto de la orientación profesional. Con este fin, se realizó una búsqueda en la base PePSIC y Scielo en agosto de 2011, a partir de criterios de selección y exclusión, y términos definidos a priori, con el alcance del análisis de un total de 24 artículos que fueron analizados en sus textos completos. Los resultados se organizan en tres bloques, de los autores, las revistas y los aspectos metodológicos. Se tratan principalmente temas relacionados con los estados de origen de los autores, la relación entre las metas y los métodos adoptados en los artículos y la relación de causalidad evidente entre el número de publicaciones de artículos para uso profesional y aprobación de las pruebas.
\end{abstract}

Palabras-clave: orientación Professional, testes psicológicos, estúdios científicos.

\section{INTRODUÇÃO}

A história da orientação profissional sugere que a área surgiu com Frank Parsons, o pioneiro na sistematização teórico-técnica da área, a partir de seus trabalhos realizados no Vocational Bureau of Boston, nos Estados Unidos, na primeira década do século XX. Parsons propôs uma série de princípios e realizou ações encadeadas e intencionalmente ordenadas que deram origem a esse campo do saber, com objetivos diretamente ligados ao aumento da eficiência industrial, ou seja, promover a mão de obra nas industrias existentes (Sparta, 2003; Ribeiro \& Uvaldo, 2007).

O Vocational Bureau of Boston tinha o intuito de auxiliar jovens a fazer escolhas de carreira e, a partir das experiências e debates sobre problemas vocacionais, Parsons publicou o livro Choosing a Vocation (1909), que segundo Ribeiro e Uvaldo (2007), foi como um manual que visava auxiliar futuros profissionais da área, apresentando os princípios básicos da orientação vocacional e a descrição de 17 casos atendidos no Bureau. A preocupação de Parsons a respeito do trabalho se justifica pelo contexto histórico e social em que ele nasceu e cresceu, em pleno desenvolvimento do emprego assalariado que, por sua vez, era acompanhado pela exploração dos trabalhadores, insegurança e desrespeito aos direitos humanos. Ao lado disso, nessa mesma época houve o surgimento de movimentos idealistas de reforma social (Ribeiro \& Uvaldo, 2007).

Sparta (2003) ressalta que Parsons acrescentou à Orientação Profissional idéias da Psicologia e da Pedagogia, definindo, em seu livro, três passos a serem seguidos durante o processo de Orientação Profissional. Essas indicações eram 
relativas à análise das características do indivíduo, a análise das características das ocupações e o cruzamento dessas informações, idéia que influenciou o surgimento da Teoria do Traço e Fator, baseando-se no autoconhecimento e no fornecimento de informação profissional.

No Brasil, a Psicologia passpu a fazer parte das relações de trabalhos a partir da década de 20. Segundo Sparta (2003), entre 1920 e 1930 a Psicologia Diferencial e a Psicometria influenciaram fortemente a prática da Orientação Profissional, devido ao desenvolvimento de testes de inteligência, aptidões, habilidades, interesses e personalidade. Tratava-se de uma prática fortemente diretiva, na qual o orientador deveria fazer um diagnóstico e um prognóstico do orientando e, com base nesses procedimentos, indicar as ocupações mais apropriadas.

Sparta (2003) menciona que no Brasil, a Orientação Profissional teve como marco inicial a criação do Serviço de Seleção e Orientação Profissional pelo engenheiro suíço Roberto Mange em 1924. Em 1947, um avanço importante foi a criação do Instituto de Seleção e Orientação Profissional (ISOP), que reuniu técnicos e estudiosos da psicologia aplicada e que tinha como objetivo desenvolver métodos e técnicas da Psicologia Aplicada ao Trabalho e à Educação, principalmente por meio da adaptação e validação de instrumentos psicológicos estrangeiros e da criação de instrumentos brasileiros, além do atendimento ao público através do processo de Seleção e Orientação Profissional e a formação de novos especialistas da área (Sparta, 2003).

Atualmente, a Orientação Vocacional está consolidada como uma modalidade de atendimento psicológico, como explica Nascimento (2007), citando que esta é uma área que comporta várias abordagens e técnicas na atuação, além de entender que o orientando possui participação ativa. A orientação deve partir de um pressuposto teórico que seja capaz de abranger a subjetividade e a realidade do orientando, incluindo esferas sociais e do trabalho. Tal orientação, conforme Nascimento (2007), deve pressupor certa criatividade, permitir variação nas técnicas e incluir movimentação nas sessões, com atividades e intervenção do orientador, facilitando a ocorrência de mudanças consistentes.

Nesse sentido, um desafio da área é compreender como o processo de avaliação psicológica pode contribuir com a escolha profissional do orientando. Como pode-se observar, ao longo da história da OP, tanto no Brasil quanto no 
exterior, a utilização de testes tem sido bastante útil e algumas pesquisas já obtiveram dados sobre essa temática.

A análise de Rueda (2009) constatou que os instrumentos utilizados em Orientação Profissional são temas de discussão e alvo de vários estudos. Há testes que são utilizados para a pratica da Orientação Profissional que são padronizados e aprovados pelo Sistema de Avaliação de Testes Psicológicos (SATEPSI) do Conselho Federal de Psicologia (CRP), tais como o Teste de Fotos de Profissões (Berufsbilder Test, BBT), Escala de Maturidade para a Escolha Profissional (EMEP), Escala de Aconselhamento Profissional (EAP), Avaliação dos Interesse Profisionas (AIP), Questionário de Busca Auto Dirigida (SDS), entre outros. Porém, segundo Noronha e Ambiel (2006), muitas técnicas não padronizadas, tais como a colagem, tem sido utilizadas nesse contexto.

Tal constatação a respeito das técnicas não padronizadas é importante e não pode ser menosprezada, desde que os dados obtidos por meio de tais procedimentos sejam compilados aos resultados dos testes, dentro de um enquadre teórico bem estabelecido, podendo auxiliar, assim, nas previsões e tomadas de decisões, tornando-as menos inflexíveis. Portanto, entende-se, assim como Melo-Silva, Leal e Fracalozzi (2010), que muito estudo deve ser despendido para que a prática da avaliação psicológica em OP seja consolidada e possa ser trabalhada nos grupos mais diversos da população brasileira.

Nesse sentido, Sparta, Bardagi e Teixeira (2006) expõem que o campo da Orientação Vocacional possui um proveitoso desenvolvimento, especialmente no que toca ao aperfeiçoamento de instrumentos psicológicos e outros recursos técnicos, visando a implementação de processos de avaliação e intervenção cada vez mais especializados. Os autores sugerem dois modelos de avaliação psicológica em OP, ligados ao desenvolvimento histórico da área. Tais modelos são Modelo de Avaliação Psicológica Centrado no Resultado e Modelo de Avaliação Psicológica Centrado no Processo.

O primeiro deles, modelo centrado no resultado, tem como característica principal a preocupação com a definição de uma escolha profissional. Para tanto, resultados de testes psicológicos que avaliem características como inteligência, aptidões, interesses e personalidade, eram combinados com as características e ambientes ocupacionais $\mathrm{e}$, assim, os resultados dos testes eram tidos como indicadores da escolha do orientando, de forma diretiva. 
Contudo, com a diminuição no uso de testes psicológicos a partir da década de 1970, a Orientação Profissional passou a receber influências de diversas teorias, com valorização do aconselhamento psicológico não diretivo e a psicologia clínica. A partir dessa mudança, o segundo modelo, de avaliação psicológica centrada no processo, parte do pressuposto de que instrumentos de avaliação podem ou não ser usados; porém, a ênfase não está mais nos resultados dos testes, mas na aprendizagem do orientando e no autoconhecimento de suas próprias condições, possibilidades e limitações.

O presente trabalho tem como objetivo fazer um levantamento da literatura científica brasileira, publicada em bases de dados nacionais de periódicos científicos, a respeito da avaliação psicológica no contexto da orientação profissional. Outros estudos, direta ou indiretamente, fizeram esse mapeamento anteriormente e revelaram uma tendência ao estudo dos interesses profissionais (principalmente), habilidades e personalidade como os mais freqüentes na literatura científica de OP, seja em artigos, teses, dissertações ou apresentações em congressos. Outro dado interessante é que a produção se concentra predominantemente nos estados de São Paulo, Santa Catarina e Rio Grande do Sul e conta com uma média de até três autores por trabalho, chegando até 13 autores em um caso isolado. Nota-se também um aumento gradativo na quantidade de trabalhos publicados, especialmente no que toca à relatos de pesquisa (Noronha \& Ambiel, 2006; Noronha \& cols., 2006; Joly, Silva, Nunes \& Souza, 2007; Teixeira, Lassance, Silva \& Bardagi, 2007; Rueda, 2009)

Os trabalhos de Noronha e Ambiel (2006), Teixeira e cols. (2007) e MeloSilva e cols. (2010) propuseram, a partir de análises qualitativas e de conteúdo, algumas categorias que compreendessem os objetivos dos trabalhos analisados. De forma geral, essas categorias englobavam assuntos relativos à história da OP, modelos de atuação e avaliação de intervenção, formação, escolha profissional em grupos específicos e avaliação de qualidade de instrumentos. Entretanto, nenhum desses trabalhos se ateve a detalhar os procedimentos de análises utilizados nos trabalhos para se atingir tais objetivos, e este será uma das análises propostas no presente estudo.

Considerando que a área de OP está em crescente processo de produção científica (Teixeira \& cols., 2007; Rueda, 2009; Melo-Silva \& cols., 2010) e que o ano de 2011 foi instituído como o Ano Temático da Avaliação Psicológica pelo Conselho Federal de Psicologia (2011), este artigo tem como objetivo analisar 
artigos brasileiros, publicados em duas bases de dados digitais, que versem sobre as duas temáticas em questão.

\section{MÉTODO}

Materiais e procedimentos

Foi feita uma busca em duas bases de dados eletrônicas brasileiras, que agrupam grande parte das revistas científicas da área da psicologia, que são PePSIC e Scielo, no mês de agosto de 2011. Nessas bases, foram usados os seguintes termos para busca dos artigos: Orientação profissional, Orientação vocacional, interesses profissionais e escolha profissional.

Para a seleção dos artigos, foram estipulados alguns critérios. Para inclusão, o artigo deveria ser de pesquisa empírica, com dados coletados no Brasil e com uso de testes ou instrumentos de avaliação psicológica padronizados, já publicados ou em construção. Como critério de exclusão, instituiu-se artigos de relato de experiências, estudos de caso e que usassem questionários ou entrevistas semi estruturadas não padronizados.

Assim, um total de 24 artigos cumpriram a todos os critérios e esse foi o $\mathrm{N}$ deste trabalho. Entretanto, alguns artigos foram selecionados por meio de mais de um critério (por exemplo, um mesmo artigo que estava indexado nas duas bases de dados) e em algumas situações esse $\mathrm{N}$ foi alterado, sendo justificado quando necessário. No Anexo 1 é possível apreciar as referências de todos os artigos analisados.

As variáveis investigadas foram quantidade de autores, instituições de origem dos autores, estados das instituições, termo que identificou o artigo, base de dados de origem do artigo, ano de publicação, revista, objetivos, testes utilizados, construtos avaliados, se os testes utilizados estavam no momento da consulta aprovados ou não pelo SATEPSI, quantidade de sujeitos da pesquisa, médias e desvios padrão das idades dos participantes, região da coleta de dados da pesquisa e análises de dados utilizadas. As informações foram buscadas nos resumos e quando não foi possível encontrá-las, procedeu-se a consulta ao texto completo.

\section{RESULTADOS}

Os resultados serão apresentados em três blocos. Inicialmente, serão descritas as informações dos autores, como quantidade, instituições e estados de 
origem. Em seguida serão apresentados os dados referentes à publicação, ou seja, termos a partir dos quais os artigos foram recuperados, em quais bases de dados, as revistas e ano de publicação. Por fim, serão apresentados os dados metodológicos, que envolvem os objetivos, testes utilizados, situação dos testes perante o SATEPSI, construtos avaliados, quantidade de sujeitos, média de idade, região onde foi feita a coleta e análises de dados.

Com relação ao primeiro bloco de informações, observou-se que a quantidade de autores por artigo variou entre 1 e 7, com média de 2,9 autores por artigo. Quanto às instituições e estados de origem, as representadas foram Universidade São Francisco (Itatiba/SP), Universidade Federal do Rio Grande do Sul (Porto Alegre/RS), Universidade Luterana do Brasil (Santa Maria/RS), Universidade de São Paulo (Ribeirão Preto/SP), Pontifícia Universidade Católica (Curitiba/PR), Universidade Presbiteriana Mackenzie (São Paulo/SP), Universidade de Passo Fundo (Passo Fundo/RS), Universidade Estadual de Londrina (Londrina/PR), Universidade Ibirapuera (São Paulo/SP), Centro Universitário Avantis (Balneário Camboriú/SC) e Editora Vetor (São Paulo/SP).

Quanto às palavras-chave, a maioria dos artigos $(n=16)$ foram recuperados pelo termo "Orientação profissional", enquanto sete artigos responderam à "Interesses Profissionais" e outros cinco por "Orientação Vocacional", totalizando 28 palavras-chave. O termo "escolha profissional" não retornou nenhum registro. O total indica que três artigos foram recuperados por meio de duas palavraschaves, sendo dois selecionados tanto por "orientação profissional" quanto por "interesses profissionais", e um respondendo à "orientação vocacional" e "interesses profissionais".

Com relação às bases de dados, 15 artigos foram selecionados na PePSIC, enquanto 10 foram recuperados na Scielo. Os artigos recuperados foram publicados entre 2000 e 2011, embora não se tenha colocado qualquer tipo de condição quanto à essa variável. Pode-se observar que em 2000 e 2002 houve uma publicação em cada ano; em 2003 e 2004, duas publicações em cada; três em 2005; cinco em 2008; sete em 2009; duas em 2010 e outras duas em 2011. Percebe-se um aumento da quantidade de publicações ao longo da década, com um decréscimo nos dois últimos anos. Provavelmente isso se explique pela demora do processo de disponibilização dos acervos nas bases de dados digitais. A Tabela 1 mostra as revistas em que os artigos recuperados foram publicados. 
Tabela 1.

Revistas que publicaram os artigos selecionados.

\begin{tabular}{cc}
\hline Revista & Frequencia \\
\hline Revista Brasileira de Orientação Profissional & 6 \\
Psicologia Escolar e Educacional & 3 \\
Psico-USF & 3 \\
Psicologia: ciência e profissão & 2 \\
Estudos de Psicologia (Campinas) & 2 \\
Psicologia: Reflexão e Crítica & 2 \\
Aletheia & 1 \\
Avaliação Psicológica & 1 \\
Boletim de Psicologia & 1 \\
Estudos de Psicologia (Natal) & 1 \\
Paidéia & 1 \\
Psic & 1 \\
Psicologia em Estudo & 1 \\
\hline
\end{tabular}

A Tabela 1 evidencia que duas das três revistas com maiores frequencias são de áreas específicas, quais sejam, de orientação profissional e de psicologia escolar e educacional. As demais revistas, com exceção de Avaliação Psicológica, são revistas com políticas editorias com tendência à pluralidade de assuntos.

A seguir, serão apresentados os dados metodológicos dos artigos estudados. Com relação aos objetivos, observou-se que os artigos propunham quatro categorias de investigação, que estão descritas na Tabela 2. A coluna "termos indicativos" exibe a forma com que o objetivo foi expresso no resumo.

A Tabela 2 informa que a maioria dos estudos tem o objetivo de verificar as correlações entre construtos, sendo que as diferenças entre grupos também são usadas em $20 \%$ dos estudos. Destaca-se que a construção de instrumentos bem como a verificação de propriedades psicométricas também se fizerem notar. Assim, buscando verificar como os objetivos foram operacionalizados no estudo, verificou-se os métodos de análises empregadas nos estudos (Tabela 3).

A Tabela 3 mostra que a análise mais utilizada foi a Correlação de Pearson, de forma concordante com os objetivos traçados. Além disso, as análises de diferença de média também foram bastante utilizadas. A Tabela 4 informa sobre os testes e instrumentos utilizados nos estudos, bem como os construtos avaliados e situação perante o SATEPSI em agosto de 2011. 
Tabela 2.

Objetivos dos estudos analisados.

\begin{tabular}{ccc}
\hline Categoria & Termos indicativos & Frequencia \\
\hline $\begin{array}{c}\text { Relação entre } \\
\text { construtos }\end{array}$ & $\begin{array}{c}\text { "analisar as relações entre"; "buscou as relações } \\
\text { entre"; "analisar as relações entre"; } \\
\text { "correlacionar"; "explorar as correlações"; } \\
\text { "investigou as correlações" }\end{array}$ & 10 \\
$\begin{array}{c}\text { Diferenças entre } \\
\text { grupos }\end{array}$ & $\begin{array}{c}\text { "analisar diferenças de média"; "analisar as } \\
\text { diferenças de médias"; "investigar em função do } \\
\text { gênero"; "investigar diferenças"; "verificar se } \\
\text { existem diferenças significativas" }\end{array}$ & 5 \\
$\begin{array}{c}\text { Construção de } \\
\text { instrumentos }\end{array}$ & $\begin{array}{c}\text { "Apresenta-se o desenvolvimento de um } \\
\text { inventário"; "visou a construção e validação de } \\
\text { uma escala"; "relata a construção" }\end{array}$ & 3 \\
$\begin{array}{c}\text { Parâmetros } \\
\text { psicométricos }\end{array}$ & $\begin{array}{c}\text { "estudar a validade concorrente"; "investigadas } \\
\text { as características psicométricas" ; "examinar } \\
\text { evidências empíricas de adequação e de } \\
\text { estabilidade dos dados normativos" }\end{array}$ & 3 \\
Caracterização \\
de amostra & $\begin{array}{c}\text { "avaliar características sócio-demográficas e } \\
\text { vocacionais"; "caracterizar e analisar a estrutura } \\
\text { de inclinação profissional"; "investigação do } \\
\text { perfil" }\end{array}$ & 3 \\
$\begin{array}{c}\text { Avaliação de } \\
\text { intervenção }\end{array}$ & $\begin{array}{c}\text { "avaliou o impacto da intervenção"; "avaliar a } \\
\text { efetividade de um programa de Orientação }\end{array}$ & 2 \\
\hline
\end{tabular}

Tabela 3.

Métodos de análise adotados.

\begin{tabular}{lccc}
\hline \multicolumn{1}{c}{ Tipo } & Análise & Frequencia & Frequencia total \\
\hline Correlação & Correlação de Pearson & 12 & \\
& Correlação de Spearman & 1 & 14 \\
Diferenças de médias & Regressão & 1 & \\
& ANOVA & 8 & \\
& Teste t & 5 & 17 \\
Precisão & Teste de Mann-Whitney & 2 & \\
Provas post hoc & Qui-quadrado & 2 & 4 \\
Redução de dados & Alfa de Cronbach & 4 & 3 \\
Qualitativa & Prova de Tukey & 3 & 2 \\
& Análise fatorial & 2 & 1 \\
\hline
\end{tabular}


Tabela 4.

Frequencia e situação dos testes utilizados.

\begin{tabular}{|c|c|c|c|}
\hline Teste & Frequência & Construto & $\begin{array}{l}\text { SATEPSI (em } \\
\text { Agosto/2011) }\end{array}$ \\
\hline $\begin{array}{l}\text { Questionário de Busca } \\
\text { Autodirigida (SDS) }\end{array}$ & 9 & Interesses profissionais & Favorável \\
\hline $\begin{array}{l}\text { Escala de Aconselhamento } \\
\text { Profissional (EAP) }\end{array}$ & 7 & Interesses profissionais & Favorável \\
\hline $\begin{array}{l}\text { Bateria de Provas de Raciocínio } \\
\text { (BPR-5) }\end{array}$ & 4 & Inteligência & Favorável \\
\hline $\begin{array}{l}\text { Teste de Fotos de Profissões } \\
\text { (BBT-Br) }\end{array}$ & 4 & Interesses profissionais & Favorável \\
\hline $\begin{array}{c}\text { Escala de Auto-eficácia para } \\
\text { Atividades Ocupacionais (EAAOc) }\end{array}$ & 3 & $\begin{array}{l}\text { Autoeficácia para } \\
\text { atividades ocupacionais }\end{array}$ & Não consta \\
\hline Escala de Indecisão Vocacional & 3 & Exploração vocacional & Não consta \\
\hline $\begin{array}{l}\text { Levantamento de Interesses } \\
\text { Profissionais (LIP) }\end{array}$ & 3 & Interesses profissionais & Não consta \\
\hline $\begin{array}{c}\text { Questionário de Personalidade } \\
16 \mathrm{PF}\end{array}$ & 3 & Personalidade & Desfavorável \\
\hline $\begin{array}{l}\text { Bateria Fatorial de Personalidade } \\
\text { (BFP) }\end{array}$ & 2 & Personalidade & Favorável \\
\hline $\begin{array}{l}\text { Escala de Maturidade para Escolha } \\
\text { profissional (EMEP) }\end{array}$ & 2 & $\begin{array}{l}\text { Maturidade para escolha } \\
\text { profissional }\end{array}$ & Favorável \\
\hline $\begin{array}{l}\text { Escala de Autoeficácia para } \\
\text { Escolha Profissional (EAE-EP) }\end{array}$ & 1 & $\begin{array}{l}\text { Autoeficácia para escolha } \\
\text { profissional }\end{array}$ & Em avaliação \\
\hline $\begin{array}{c}\text { Escala de Entrincheiramento na } \\
\text { Carreira (EEC) }\end{array}$ & 1 & $\begin{array}{l}\text { Entricheiramento de } \\
\text { carreira }\end{array}$ & Não consta \\
\hline $\begin{array}{l}\text { Inventário Fatorial de } \\
\text { Personalidade (IFP) }\end{array}$ & 1 & Personalidade & Favorável \\
\hline $\begin{array}{c}\text { Inventário de Levantamento das } \\
\text { Dificuldades da Decisão } \\
\text { Profissional (IDDP) }\end{array}$ & 1 & $\begin{array}{l}\text { Dificuldades de decisão } \\
\text { profissional }\end{array}$ & Não consta \\
\hline Inventário de Interesses Angelini & 1 & Interesses profissionais & Não consta \\
\hline $\begin{array}{l}\text { Inventário Brasileiro de } \\
\text { Desenvolvimento Profissional }\end{array}$ & 1 & $\begin{array}{l}\text { Desenvolvimento } \\
\text { profissional }\end{array}$ & Não consta \\
\hline $\begin{array}{c}\text { Questionário de Vivências } \\
\text { Acadêmicas - reduzido (QVA-r) }\end{array}$ & 1 & Vivências acadêmicas & Não consta \\
\hline Bem Sex Role Inventory - BSRI & 1 & Papéis de gênero & Não consta \\
\hline $\begin{array}{l}\text { Inventário de Satisfação do } \\
\text { Consumidor }\end{array}$ & 1 & Satisfação do consumidor & Não consta \\
\hline $\begin{array}{c}\text { Matrizes Progressivas de Raven - } \\
\text { Escala geral }\end{array}$ & 1 & Inteligência & Favorável \\
\hline
\end{tabular}


Como se vê, entre os quatro testes mais utilizados, três são de avaliação de interesses profissionais, a partir de perspectivas teóricas diferentes. Além disso, os testes mais utilizados têm parecer favorável do SATEPSI.

A respeito dos participantes, no total os estudos contaram com dados de 5519 pessoas, com média de 229,96 participantes por estudo, com variação de 59 a 950 pessoas. As idades variaram entre 13 e 59 anos, com média de idade de 19,3 anos e desvio-padrão médio de 3,1. Os dados coletados foram provenientes dos estados de São Paulo (15 artigos), Paraná (4 artigos) e Rio Grande do Sul (2 artigos), sendo que em quatro artigos essa informação não foi encontrada.

\section{DISCUSSÃO}

Considerando que o objetivo de estudos de levantamento de literatura é mapear a produção científica em uma área ou tema específico, este artigo objetivou expor algumas características de pesquisas brasileiras sobre avaliação psicológica no contexto da orientação profissional. Dessa forma, a própria intenção deste trabalho é original, uma vez que outros artigos visaram levantar o estado da arte da Orientação Profissional de forma geral, a partir a análises da Revista Brasileira de Orientação Profissional (Teixeira \& cols., 2007; Rueda, 2009), de trabalhos apresentados em congressos (Melo-Silva \& cols., 2010) ou em bases de dados de teses, dissertações e artigos (Noronha \& cols., 2006; Noronha \& Ambiel, 2006). Mesmo não tendo focado especificamente nas questões relativas à avaliação psicológica, temáticas relativas à esse assunto foram presentes em todos os artigos citados, bem como a OP foi verificada em estudo sobre trabalhos apresentados em congressos de avaliação psicológica (Joly \& cols., 2007).

Pode-se perceber por esses dados, bem como pela análise da história da OP no Brasil e em outros países (Sparta, 2003; Ribeiro \& Uvaldo, 2007) que a avaliação psicológica tem sido bastante utilizada em estudos científicos, bem como na prática dos profissionais. Sparta e cols. (2006) definem que, em determinado momento da história de evolução da área, o foco do trabalho era nos resultados dos testes, algo que foi sendo flexibilizado, chegando-se a compreensão de que o orientando deve ter uma postura essencialmente ativa (Nascimento, 2007). 
Com relação aos achados do presente estudo, os resultados mostraram que o termo que mais identificou artigos foi Orientação Profissional, seguido por Interesses profissionais e Orientação Vocacional. Essa situação envolve uma antiga questão da área, que parece ainda não ter uma resolução adequada, que é a respeito da nomenclatura, especialmente quanto ao uso de "profissional" ou "vocacional", em complemento à "orientação". Uma outra questão que deve ser abordada é sobre a escolha do termo "interesses profissionais". Diferente dos demais termos, que buscam denominar uma área, este termo designa um construto, o que pode ter enviesado os resultados no que diz respeito aos assuntos mais estudados nos artigos. Entretanto, historicamente, os interesses profissionais são os construtos mais estudados e aplicados na práticas dos orientadores (Noronha \& Ambiel, 2006; Sparta \& cols., 2006) e, dessa forma, optou-se por incluir a fim de se aumentar o escopo do trabalho.

Apesar da grande historia de uso dos testes em OP, chama a atenção o fato de que foram localizados artigos, segundo os critérios predefinidos, datados apenas a partir de 2000, com uma prevalência da base de dados PePSIC em relação à Scielo. Uma hipótese que pode ser levantada a esse respeito é de que esses dados podem estar relacionados ao próprio crescimento da área que, conforme apontaram estudos anteriores, especialmente de Noronha e Ambiel (2006) e Teixeira e cols. (2007), tiveram um ápice a partir de 2000, após ao menos duas décadas de pouca produção. Além disso, um grande número de revistas foram fundadas na década passada, além das próprias bases de dados. Contudo, deve-se considerar que algumas revistas disponibilizam seus acervos mais antigos também de forma digital nas bases pesquisadas e, ainda assim, apenas uma produção relativamente recente pode ser recuperada.

Em relação aos autores e suas origens geográficas, diferentemente do que foi encontrado por Noronha e Ambiel (2006), percebe-se uma predominância de trabalhos multiautorais. Entretanto, conforme outros artigos demonstraram, existe uma predominância muito marcada de autores das regiões sudeste e sul, principalmente dos estados de São Paulo, Paraná, Santa Catarina e Rio Grande do Sul, com nenhuma participação das demais regiões. Frente a esses dados é primordial que se indague o por que disso. Seria uma questão de investimento por parte das universidades? Ou uma demanda que não chega aos psicólogos das regiões não representadas? Independente de qual seja a resposta, se faz urgente que mais pesquisas sejam desenvolvidas para que, entre outros 
objetivos, os testes psicológicos usados em OP possam também conter estudos de validade e normas para todo o Brasil, de uma forma mais distribuída ao longo de todo o território.

Quanto aos quesitos metodológicos analisados, percebe que a maioria dos trabalhos buscou realizar estudos correlacionais e que investigassem diferenças entre certos grupos. Por outro lado, apenas dois trabalhos tiveram o objetivo de aferir a qualidade de intervenções. Ao verificar as estratégias de análise utilizadas, verifica-se a coerência no caminho percorrido entre o objetivo e a obtenção dos resultados, bem como na utilização de amostras relativamente grandes nos estudos, o que é uma característica bastante presente em estudos de cunho quantitativo.

Vale um comentário sobre os testes mais utilizados. Dentre dos quatro testes mais utilizados, três são de avaliação de interesses profissionais e um de inteligência, mas todos têm o parecer favorável do SATEPSI. Esse resultado é digno de nota, uma vez que não se dá por acaso, mas por haver uma relação um causal entre a preocupação com a qualidade científica dos instrumentos, a quantidade de estudos realizados com eles e o fato de estarem aprovados para uso profissional. Esse dado deve servir de incentivo aos autores dos instrumentos que também aparecem na tabela, especialmente àqueles que tiveram seus dados relatados em estudos de construção, mas que ainda não constam no processo avaliativo do SATEPSI.

\section{CONSIDERAÇÕES FINAIS}

Considerando a avaliação psicológica como uma área de atuação transversal da psicologia e as discussões fomentadas pelo Ano Temático instituído pelo CFP, este estudo contribuiu para uma compreensão do tema a partir da temática da orientação profissional. Deve-se ressaltar que o intuito não foi fazer um levantamento sistemático do assunto, mas a opção por manter-se apenas em duas bases de dados (PePSIC e Scielo) se deu por serem essas duas bases de dados nacionais, embora agrupem também publicações estrangeiras.

Espera-se que, com esse artigo, se tenha contribuído para indicar caminhos futuros para pesquisas na área, tais como construtos que carecem de mais pesquisas e, especialmente, metodologias de avaliação de processos de OP. Tendo em vista a grande contribuição mútua que se observa tanto na história da avaliação psicológica quanto da orientação profissional, o reconhecimento da 
psicologia e de suas áreas de atuação na sociedade passa pela ciência, principalmente quando esta consegue identificar lacunas a serem preenchidas a partir de demandas sociais.

\section{REFERÊNCIAS}

Joly, M. C. A. J., Silva, M. C. R., Nunes, M. F. O., \& Souza, M. S. (2007). Análise da produção científica em painéis dos Congressos Brasileiros de Avaliação Psicológica. Avaliação Psicológica, 6(2), 239-252.

Melo-Silva, L. L.; Leal, M. S., \& Fracalozzi, N. M. N. (2010). Produção científica em congressos brasileiros de orientação vocacional e profissional: Período 1999-2009. Revista Brasileira de Orientação Profissional, 11(1), pp. 107120.

Nascimento, R. S. G. F. (2007). Avaliação psicológica em processos dinâmicos de orientação vocacional individual. Revista Brasileira de Orientação Profissional, 8(1), 33-44.

Noronha, A. P. P., \& Ambiel, R. A. M. (2006). Orientação profissional e vocacional: análise da produção científica. Psico-USF, 11(1), pp. 75-84.

Noronha, A. P. P., Andrade, R. G., Miguel, F. K., Nascimento, M. M., Nunes, M. F. O., Pacanaro, S. V., \& cols. (2006). Análise de teses e dissertações em orientação profissional. Revista da $A B O P, 7(2), 1-10$.

Ribeiro, M. A., \& Uvaldo, M. C. C. (2007). Frank Parsons: Trajetória do pioneiro da orientação vocacional, profissional e de carreira. Revista Brasileira de Orientação Profissional, 8(1), 19-31.

Rueda, F. J. M. (2009). Produção científica da Revista Brasileira de Orientação Profissional. Revista de Orientação Profissional, 10(2), 129-139.

Sparta, M. (2003). O Desenvolvimento da Orientação Profissional no Brasil. Revista Brasileira de Orientação Profissional, 4(1/2), 1-11.

Sparta, M., Bardagi, M. P., \& Teixeira, M. A. P. (2006). Modelos e Instrumentos de Avaliação em orientação profissional: perspectiva histórica e situação no Brasil. Revista Brasileira de Orientação Profissional, 7(2), 19-32.

Teixeira, M. A. P., Lassance, M. C. P., Silva, B. M. B., \& Bardagi, M. P. (2007). Produção científica em orientação profissional: uma análise da Revista Brasileira de Orientação Profissional. Revista Brasileira de Orientação Profissional, 8(2), 25-40. 
Contato: rodolfo.ambiel@usf.edu.br,m_polli@hotmail.com

Recebido em: 20/01/2011

Revisado em: 12/04/2011

Aceito em: 29/04/2011 


\section{ANEXO 1}

Neste anexo, estão relacionados os artigos que atenderam aos critérios de seleção estabelecidos e que compuseram o corpo de análise deste artigo:

Ambiel, R. A. M., \& Noronha, A. P. P. (2011). Construção dos itens da Escala de Autoeficácia para Escolha Profissional. Psico-USF, 16(1), 23-32. Recuperado em 26 de agosto de 2011 de http://www.scielo.br/pdf/pusf/v16n1/a04v16n1.pdf

Bueno, J. M. H., Lemos, C. G., \& Tomé, F. A. M. F. (2004). Interesses profissionais de um grupo de estudantes de psicologia e suas relações com inteligência e personalidade. Psicologia em Estudo (Maringá), 9(2), 271278. Recuperado em 26 de agosto de 2011, http://www.scielo.br/pdf/pe/v9n2/v9n2a13.pdf

Godoy, S., Noronha, A. P. P., Ambiel, R. A. M., \& Nunes, M. F. O. (2008). Instrumentos de inteligência e interesses em orientação profissional. Estudos de Psicologia, 13(1), 75-81. Recuperado em 26 de agosto de 2011 de http://www.scielo.br/pdf/epsic/v13n1/09.pdf

Lassance, M. C. P., Bardagi, M. P., \& Teixeira, M. A. P. (2009). Avaliação de uma intervenção cognitivo-evolutiva em orientação profissional com um grupo de adolescentes brasileiros. Revista Brasileira de Orientação Profissional, 10(1), 23-32. Recuperado em 26 de agosto de 2011, de http://pepsic.bvsalud.org/pdf/rbop/v10n1/v10n1a05.pdf

Lobato, C. R. P. S., \& Koller, S. H. (2003). Maturidade Vocacional e Gênero: Adaptação e Uso do Inventário Brasileiro de Desenvolvimento Profissional. Revista Brasielira de Orientação Profissional, 4(1/2), 57-69. Recuperado em 26 de agosto de 2011, de http://pepsic.bvsalud.org/pdf/rbop/v4n1-2/v4n12a06.pdf

Magalhães, M. O. (2008). Propriedades psicométricas da versão brasileira da Escala de Entrincheiramento na Carreira. Psico-USF, 13(1), 13-19. Recuperado em 26 de agosto de 2011 de www.scielo.br. 
Magalhães, M. O. (2008). Relação entre ordem de nascimento e interesses vocacionais. Estudos de Psicologia (Campinas), 25(2) 203-210. Recuperado em 26 de agosto de 2011, de http://www.scielo.br/pdf/estpsi/v25n2/a05v25n2.pdf

Melo-Silva, L. L., Noce, M. A., \& Andrade, P. P. (2003). Interesses em adolescentes que procuram orientação profissional. PSIC - Revista de Psicologia da Vetor Editora, 4(2), 6-17. Recuperado em 26 de agosto de 2011, de http://pepsic.bvsalud.org/pdf/psic/v4n2/v4n2a02.pdf

Moura, C. B., Sampaio, A. C. P., Gemelli, K. R., Rodrigues, L. D., \& Menezes, M. V. (2005). Avaliação de um Programa Comportamental de Orientação Profissional para Adolescente. Revista Brasileira de Orientação Profissional, 6(1), 25-40. Recuperado em 26 de agosto de 2011, de http://pepsic.bvsalud.org/pdf/rbop/v6n1/v6n1a04.pdf

Neiva, K. M. C., Silva, M. B., Miranda, V. R., \& Esteves, C. (2005). Um Estudo sobre a Maturidade para a Escolha Profissional de Alunos do Ensino Médio. Revista Brasileira de Orientação Profissional, 6(1), 1-14. Recuperado em 26 de agosto de 2011, de http://pepsic.bvsalud.org/pdf/rbop/v6n1/v6n1a02.pdf

Noronha, A. P. P., \& Ottati, F. (2010). Interesses profissionais de jovens e escolaridade dos pais. Revista Brasileira de Orientação Profissional, 11(1), 37-47. Recuperado em 26 de agosto de 2011, de http://pepsic.bvsalud.org/pdf/rbop/v11n1/v11n1a05.pdf

Noronha, A. P. P., Martins, D. F., Gurgel, M. G. A., \& Ambiel, R. A. M. (2009). Estudo correlacional entre interesses profissionais e vivências acadêmicas no Ensino Superior. Revista Semestral da Associação Brasileira de Psicologia Escolar e Educacional (ABRAPEE), 13(1), 143-154. Recuperado em 26 de agosto de 2011, de http://www.scielo.br/pdf/pee/v13n1/v13n1a16.pdf

Nunes, M. F. O., \& Noronha, A. P. P. (2008). Escala de Auto-eficácia para Atividades Ocupacionais: Construção e estudos exploratório. Paidéia, 18(39), 111-12. Recuperado em 26 de agosto de 2011, de http://www.scielo.br/pdf/paideia/v18n39/v18n39a11.pdf

Nunes, M. F. O., \& Noronha, A. P. P. (2009). Auto-Eficácia para Atividades Ocupacionais e Interesses Profissionais em Estudantes do Ensino Médio. Psicologia: Ciência e Profissão, 29(1), 102-115. Recuperado em 26 de agosto de 2011, de http://pepsic.bvsalud.org/pdf/pcp/v29n1/v29n1a09.pdf 
Nunes, M. F. O., \& Noronha, A. P. P. (2009). Relações entre interesses, personalidade e habilidades cognitivas: um estudo com adolescentes. PsicoUSF, 14(2), 131-141. Recuperado em 26 de agosto de 2011, de http://www.scielo.br/pdf/pusf/v14n2/v14n2a02.pdf

Nunes, M. F. O., \& Noronha, A. P. P. (2011). Associações entre Auto-Eficácia para Atividades Ocupacionais e Interesses em Adolescentes. Psicologia: Reflexão e Crítica, 24(1), 1-9. Recuperado em 26 de agosto de 2011 de http://www.scielo.br/pdf/prc/v24n1/v24n1a02.pdf

Pasian, S. R., \& Jardim-Maran, M. L.C. (2008). Padrões normativos do BBT-BR em adolescentes: Uma verificação da atualidade das normas disponíveis. Revista Brasileira de Orientação Profissional, 9(1), 61-74. Recuperado em 26 de agosto de 2011, de http://pepsic.bvsalud.org/pdf/rbop/v9n1/v9n1a06.pdf

Primi, R., Bighetti, C. A., Munhoz, A. H., Noronha, A. P. P., Polydoro, S. A. J., Di Nucci, E. P., \& Pellegrini, M. C. K. (2002). Personalidade, Interesses e Habilidades: Um estudo correlacional da BPR-5, LIP e do 16PF. Avaliação Psicológica, 1, 61-72. Recuperado em 26 de agosto de 2011, de http://pepsic.bvsalud.org/pdf/avp/v1n1/v1n1a07.pdf

Primi, R., Moggi, M. A., \& Casellato, E. O. (2004). Estudo correlacional do Inventário de Busca Autodirigida (Self-directed Search) com o IFP. Psicologia Escolar e Educacional, 8(1), 47-54. Recuperado em 26 de agosto de 2011, de http://www.scielo.br/pdf/pee/v8n1/v8n1a06.pdf

Primi, R., Munhoz, A. M. H., Bighetti, C. A., Di Nucci, E. P., Pellegrini, M. C. K., \& Moggi, M. A. (2000). Desenvolvimento de um Inventário de Levantamento das Dificuldades da Decisão Profissional. Psicologia: Reflexão e Crítica, 13(3), 451-463. Recuperado em 26 de agosto de 2011, de http://www.scielo.br/pdf/prc/v13n3/v13n3a13.pdf

Sartori, F. A., Noronha, A. P. P., \& Nunes, M. F. O. (2009). Comparações entre EAP e SDS: interesses profissionais em alunos do ensino médio. Boletim de Psicologia, LIX(130), 17-29. Recuperado em 26 de agosto de 2011, de http://pepsic.bvsalud.org/pdf/bolpsi/v59n130/v59n130a03.pdf 
Sartori, F. A., Noronha, A. P. P., Godoy, S., \& Ambiel, R. A. M. (2010). Interesses profissionais de jovens de ensino médio: estudo correlacional entre a Escala de Aconselhamento Profissional e o Self-directed Search Carrier Explorer. Estudos de Psicologia (Campinas), 27(2), 215-225. Recuperado em 26 de agosto de 2011 de http://www.scielo.br/pdf/estpsi/v27n2/a09v27n2.pdf

Sparta, M., Bardagi, M. P., \& Andrade, A. M. J. (2005). Exploração vocacional e informação profissional percebida em estudantes carentes. Aletheia, 22, 7988. Recuperado em 26 de agosto de 2011, de http://pepsic.bvsalud.org/pdf/aletheia/n22/n22a08.pdf

Welter, G. M. R., \& Capitão, C. G. (2009). Inclinação Profissional e Personalidade: Estudo de Correlação entre Medidas desses Construtos. Psicologia: Ciência e Profissão, 29(3), 588-601. Recuperado em 26 de agosto de 2011, de http://pepsic.bvsalud.org/pdf/pcp/v29n3/v29n3a12.pdf 